\title{
PAPER
}

\section{Effect of a multidisciplinary amyotrophic lateral sclerosis (ALS) clinic on ALS survival: a population based study, 1996-2000}

\author{
B J Traynor, M Alexander, B Corr, E Frost, O Hardiman
}

Correspondence to: Dr Traynor, Massachusetts General Hospital East, Cecil B. Day Lab, Building 114, Room 3125, 16th Street, Charlestown, MA 02129 , USA;

btraynor@partners.org

Received

20 October 2002

In revised form

3 January 2003

Accepted

10 January 2003

Background: In recent years, there has been a paradigm shift in the method of healthcare delivery to amyotrophic lateral sclerosis (ALS) patients with the emergence of multidisciplinary ALS clinics that cater exclusively for patients with this condition. The impact of multidisciplinary management has not been previously evaluated.

Methods: Using data from the Irish ALS Register, we conducted a prospective, population based study of all ALS cases diagnosed in Ireland over a five year period to evaluate the effectiveness of a multidisciplinary clinic on ALS survival.

Results: Eighty two (24\%) patients attended the multidisciplinary ALS clinic, with the remaining 262 $(76 \%)$ cases followed in a general neurology clinic. The ALS clinic cohort was an average of five years younger $(60.1 \vee 65.6$ years) and were more likely to receive riluzole than the general neurology cohort (99\% v 61\%). The median survival of the ALS clinic cohort was 7.5 months longer than for patients in the general neurology cohort (logrank $=15.4, p<0.0001$ ). Overall, one year mortality was decreased by $29.7 \%$. Prognosis of bulbar onset patients was extended by 9.6 months if they attended the ALS clinic. Using multivariate analysis, attendance at the ALS clinic was an independent covariate of survival ( $H R=1.47, p=0.02)$.

Conclusions: ALS patients who received their care at a multidisciplinary clinic had a better prognosis than patients attending a general neurology clinic. The data suggest that active and aggressive management enhances survival, particularly among ALS patients with bulbar dysfunction. The effect of clinic type must be considered in future clinical trials design.

A myotrophic lateral sclerosis (ALS) is a neurodegenerative disorder affecting motor neurons, characterised by paralysis and death within three to five years from the time of diagnosis. ${ }^{1}$ ALS is currently incurable and management of affected individuals is essentially symptom based. ${ }^{2}$ In recent years, there has been a paradigm shift in the method of healthcare delivery to ALS patients: multidisciplinary ALS clinics have emerged that exclusively cater to patients with this condition. ${ }^{3}$ The concentrating of large numbers of patients in ALS clinics leads to an accumulation of resources and clinical expertise that facilitates the management of this rapidly progressive disease. Although multidisciplinary clinics improve quality of life and prolong survival in other neurodegenerative diseases, ${ }^{4}$ the effect of such clinics on ALS survival is unknown.

A multidisciplinary clinic that specialises in the diagnosis, management, and palliative care of a large number of ALS patients has been in operation in Ireland since $1995 .{ }^{5}$ We evaluated data from the Irish ALS Register to determine whether ALS patients attending this clinic over the five year period from 1996-2000 had an improved prognosis compared with other affected individuals who received their medical care in non-specialist, general neurology clinics.

\section{METHODS}

\section{The Irish ALS Register}

The strength of this study lies in the existence of a complete register of all patients diagnosed with ALS in the Republic of Ireland since 1993. ${ }^{5}$ Multiple sources of information were used in order to ensure complete case ascertainment. Details of the clinical features and medical care provided to residents of the entire community were available for study. A detailed attempt was made to exclude patients with ALS mimic syndromes. The
Irish ALS Register did not routinely collect data on functional measures-for example, vital capacity.

\section{The multidisciplinary ALS clinic}

An efficient multidisciplinary clinic has been developed that services the Irish ALS population by combining the existing infrastructure of community services and the services of a voluntary organisation (Irish Motor Neurone Disease Association (IMNDA)) with a hospital based specialist clinic. ${ }^{6}$ The clinic was based at the national neuroscience centre of Ireland. It was staffed by a core multidisciplinary team including neurologists, specialist nurses, physical, occupational, and speech therapists, and a pulmonologist, nutritionist, psychologist, and social worker. A representative from the IMNDA also attended the clinic. All services, including the equipment (such as hospital beds), were provided at no cost to the patient.

Patients were reviewed approximately every six weeks and were contacted by telephone at least once per month. Patients in the terminal stages of the illness, who could no longer attend the clinic, were visited in their homes by a specialist ALS nurse. Palliative care was provided jointly by the ALS clinic staff and local hospice home care services. A flexible approach to management was maintained throughout and emphasis was placed on early referral for clinical assessment, prompt intervention and assiduous follow up within the community.

Abbreviations: ALS, amyotrophic lateral sclerosis; IMNDA, Irish Motor Neurone Disease Association; NIPPV, non-invasive positive pressure ventilation 
The diagnosis was established according to well accepted criteria $^{7}$ and information was communicated to the patient by the senior clinician, with a family member present. ${ }^{8}$ Management of ALS patients was according to the best published practice. $^{9}$

Nutritional status and bulbar function were closely monitored: all patients were reviewed at 6-12 week intervals by a specialist nutritionist and speech and swallow therapist. Patients were informed early in their disease course of the benefits of early gastrostomy placement. Criteria for gastrostomy insertion included weight loss greater than $5 \%$ of baseline body mass ${ }^{10}$ and/or declining forced vital capacity. ${ }^{11}$

An increasing number of Irish ALS patients opted for a trial of non-invasive positive pressure ventilation (NIPPV). Approximately half of eligible patients were unable to tolerate this intervention. Of those who continued to use NIPPV, none had progressed to full mechanical ventilation. The Irish public health system does not fund either home based NIPPV or chronic mechanical ventilation for ALS patients. In mid 1999, IMNDA instituted funding for ventilator equipment, personnel, and maintenance.

Riluzole was available free of charge to Irish ALS patients provided a consultant neurologist prescribes it. There were no restrictions on the early prescription of the drug.

The structure of Irish society and its health service, and strong sense of community within Ireland dictate that the majority of patients were cared for by their families and general practitioners, and consequently most patients (60\%) died at home. ${ }^{6}$ The counselling service provided by the liaison nurse increased dramatically during this time. This service aimed to prevent crisis admissions to hospital in the agonal phases of the disease, thus reducing the risks of unplanned emergency ventilation.

\section{General neurology clinics}

There were nine general neurology clinics in operation in Ireland during the study period, staffed by neurologists whose primary interest was not ALS. ALS patients attending these clinics were reviewed less frequently than patients attending the multidisciplinary clinic-for example, biannually. These clinics were not staffed by ancillary service professionals, or by a liaison from the IMNDA. Pulmonary function tests were not routinely evaluated. Consequently, less attention was paid to early introduction of gastrostomy feeding or NIPPV and there was no well defined pathway for rapid hospital admission for urgent intervention or palliative care.

\section{Data analysis}

Data were stored on a computerised database, ${ }^{12}$ allowing for organised retrieval of data. Statistical analyses were performed using statistical software. ${ }^{13}$ Survival modeling was performed using Kaplan-Meier analysis ${ }^{14}$ and differences in survival were measured by the logrank sum test. Multivariate analysis of risk used the Cox proportional hazard model. ${ }^{15}$ Calculations were performed by using the date of diagnosis as day 0 and the primary endpoint was tracheotomy-free survival. Date of last follow up of patients was 1 July 2001. Statistical significance implied a p value $<0.05$ unless otherwise stated.

A patient was defined as an attendee at the ALS clinic if they were reviewed on more than two occasions and the first visit occurs within one year of the time of diagnosis. Date of diagnosis represented the date on which the patient was informed of their diagnosis. Delay in diagnosis was the period between symptom onset and establishment of the diagnosis of ALS.

\section{RESULTS}

During the five year study period between 1 January 1996 and 31 December 2000, 345 Irish residents were diagnosed as having suspected, possible, probable, or definite ALS. Of these, 82
Table 1 Demographics of Irish ALS patients attending multidisciplinary and general neurology clinics, 1996-2000

\begin{tabular}{|c|c|c|}
\hline & $\begin{array}{l}\text { General neurology } \\
\text { clinics }\left(n=262^{*}\right)\end{array}$ & $\begin{array}{l}\text { Multidisciplinary } \\
\text { clinic }\left(\mathrm{n}=82^{*}\right)\end{array}$ \\
\hline Mean age (range, years) ${ }^{\dagger}$ & $65.6(25.0-92.1)$ & 60.1 (19.7-83.0) \\
\hline Gender ratio $(F: M)^{\ddagger}$ & $115: 147$ & $35: 47$ \\
\hline Family history of ALS $\$$ & $7(2.7 \%)$ & $10(12.2 \%)$ \\
\hline Generalised onset ${ }^{\pi}$ & $38(14.5 \%)$ & $6(7.4 \%)$ \\
\hline Bulbar onset ${ }^{\Uparrow}$ & $107(40.8 \%)$ & $28(34.1 \%)$ \\
\hline Limb onset" & $117(44.7 \%)$ & $48(58.5 \%)$ \\
\hline $\begin{array}{l}\text { Delay in diagnosis } \\
\text { (range, days)** }\end{array}$ & 308 (29-3287) & $396(30-2188)$ \\
\hline Riluzole use & $149(60.6 \%)$ & $80(98.8 \%)$ \\
\hline NIPPV $\ddagger \ddagger$ & $7(2.7 \%)$ & $5(6.1 \%)$ \\
\hline
\end{tabular}

*excludes 1 patient with missing data

$\dagger t$ test $=-3.6, p=0.0003$

$\ddagger \chi^{2}=0.04, p=0.85$

$\S$ Fisher exact test $p=0.0016$

$\mathbb{q} \chi^{2}=5.7, p=0.06$

**Mann-Whitney $p=0.56$

$\dagger †$ missing data $=17$ patients, $\chi^{2}=42.4, p<0.00$

$\ddagger \ddagger \chi^{2}=2.17, p=0.14$

(24\%) patients routinely attended the multidisciplinary ALS clinic (ALS clinic cohort). The remaining 262 (76\%) patients attended one of nine general neurology clinics (general neurology clinic cohort). Data were missing for one patient $(0.3 \%)$. Mean follow up time was 1.5 years (range, 0.0 to 5.4 ). Five ALS clinic and seven general clinic patients received NIPPV during the current study.

The demographics of the two cohorts are shown in table 1. When comparing the two groups, there was a higher percentage of patients prescribed riluzole in the ALS clinic cohort than in the general neurology cohort (99\% v 61\%). Patients attending the ALS clinic were an average of 5.5 years younger than patients attending the general neurology clinic. Familial ALS (FALS) patients were more likely to attend the ALS clinic than a general neurology clinic ( $12 \% \vee 2 \%)$.

Figure 1 shows the survival curves of the 344 Irish ALS patients according to the type of clinic attended. Median survival of ALS patients attending the multidisciplinary clinic was 677 days compared with 448 days among individuals attending general neurology clinics (logrank $=11.6, \mathrm{p}=$ $0.0007)$. Attendance at the multidisciplinary clinic was associated with a $29.7 \%(\mathrm{z}=6.25, \mathrm{p}<0.0001)$ and $10.7 \%(\mathrm{z}=1.64$, $\mathrm{p}=0.051$ ) reduction in mortality at 12 and 24 months respectively. This beneficial effect persisted throughout the follow up period: at four years, the mortality rate of Irish ALS patients attending the ALS clinic was $13.4 \%$ lower than their counterparts attending a general neurology clinic. At the time of last follow up, 49 patients (60\%) of ALS clinic cohort were deceased, compared with 175 patients $(67 \%)$ in the general neurology clinic group.

The median survival of bulbar onset patients who received their medical care at the ALS clinic $(\mathrm{n}=34)$ was 657 days, compared with a median survival of 363 days among similarly affected individuals attending the general neurology clinic ( $\mathrm{n}=145$, figure $2, \mathrm{p}=0.0007)$. Mortality rate was decreased by $39 \%(z=5.65, p<0.0001)$ and $18 \%(z=1.87, p=0.03)$ at one and two years respectively. In contrast, clinic type did not influence the prognosis of limb onset ALS patients $(p=0.38)$. Despite the improved outcome of patients with bulbar involvement who attended the multidisciplinary clinic, their overall survival remained shorter than patients with spinal onset ( median survival $=730$ days) .

Applying the Cox proportional hazard model, four attributes were significant independent predictors of diminished survival, namely attendance at a general neurology clinic, increasing age at diagnosis, bulbar onset disease, and a short delay in diagnosis (table 2). Attending the ALS clinic 


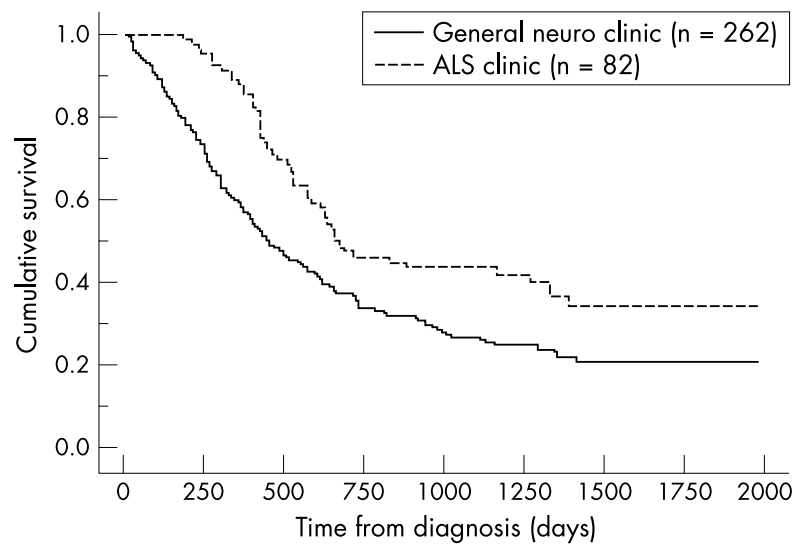

Figure 1 Survival of Irish ALS patients according to the clinic type attended, 1996-2000.

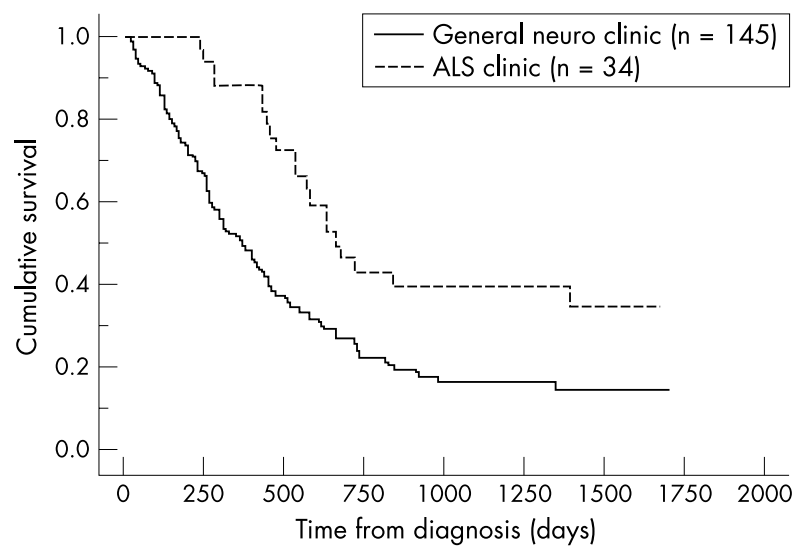

Figure 2 Survival of Irish ALS patients with bulbar onset disease according to the clinic type attended, 1996-2000.

Table 2 Cox proportional hazard model of the Irish ALS population, 1996-2000

\begin{tabular}{llll}
\hline Co-variate* $\dagger$ & $\begin{array}{l}\text { Hazard } \\
\text { ratio }\end{array}$ & $95 \% \mathrm{Cl}$ & p value \\
\hline General neurology clinic & 1.47 & $1.06-2.06$ & 0.02 \\
Bulbar onset disease & 1.41 & $1.12-1.95$ & 0.02 \\
Delay in diagnosis (per month) & 0.98 & $0.96-0.996$ & 0.02 \\
Age at diagnosis (per year) & 1.02 & $1.01-1.04$ & 0.002 \\
\hline
\end{tabular}

*non-significant covariates excluded from model include: gender, follow up time, NIPPV use, and family history of ALS. Riluzole use was not included due to dependence on the type of clinic attended. †likelihood ratio $=42.8, \mathrm{df}=4, \mathrm{p}<0.0001$

was associated with a $47.3 \%$ decreased risk for death, whereas bulbar onset disease was associated with a $40.5 \%$ increased risk of death. Data concerning riluzole use could not be included in the model, as nearly all patients attending the multidisciplinary clinic were prescribed riluzole, so that they were interdependant variables.

\section{DISCUSSION}

This is the first study to prospectively examine the effect of a multidisciplinary ALS clinic on survival. In this study, Irish ALS patients who attended a multidisciplinary ALS clinic lived an average of 7.5 months longer than individuals who received their medical care at a general neurology clinic. One year mortality was reduced by nearly $30 \%$ in patients who attended the ALS clinic. This is comparable to the reduction in one year mortality observed with riluzole in clinical trials $(38.6 \%) .{ }^{16} \mathrm{We}$

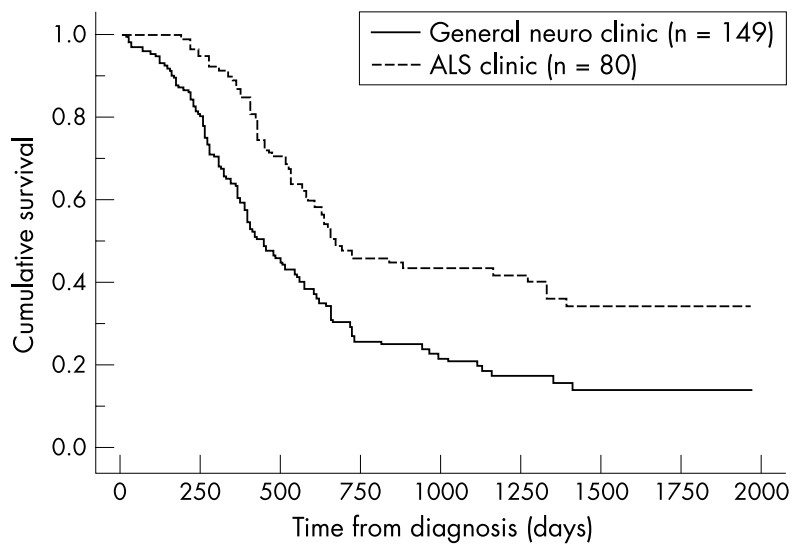

Figure 3 Survival of Irish ALS patients attending the multidisciplinary clinic compared to patients prescribed riluzole and attending a general neurology clinic, 1996-2000.

also showed that bulbar onset patients specifically benefit from attendance at a multidisciplinary ALS clinic. This is the first research to demonstrate that attendance at an ALS clinic is an independent prognostic factor in ALS survival.

A number of potential biases and confounders can be introduced by retrospectively superimposing a hypothesis on a previously collected database. ${ }^{17}$ The greater patient variability inherent in population based studies (compared with the formal inclusion and exclusion criteria of clinical trials) complicated inter-group comparison. In the current study, the demographic characteristics of the two cohorts were similar, with the exception of a five year difference in the mean age of diagnosis and a greater percentage of familial patients attending the multidisciplinary clinic. Applying the Cox model, a five year difference increased the risk of death by $10 \%$, which did not fully account for the observed differences between the cohorts. Although the median survival of familial ALS cases was generally shorter than for sporadic cases, ${ }^{18}$ the small number of cases involved ( 17 cases, 5\% of total) was unlikely to significantly alter the outcome. The similarity of the demographics and clinical features of the two cohorts suggested that neurologists did not selectively refer certain patients to the ALS clinic, though referral bias cannot be fully discounted. In order to minimise bias, patients were only considered to have attended the ALS clinic if they were reviewed on more than one occasion and their first visit was within one year of their ALS diagnosis. However, inclusion of all patients who attended the ALS clinic on only one occasion or at any stage of their illness $(n=58)$ in the ALS clinic cohort yielded very similar results (logrank $=6.1, p=0.01$, one year decrease in mortality $=25.3 \%$ ). This may indicate that attending a specialty clinic on one occasion on a consult basis, or in advanced stages of ALS, improves survival.

The higher rate of prescription of riluzole among the ALS clinic cohort compared with patients attending a general neurology clinic ( $99 \%$ v 61\%) may have biased the study. However, we have previously shown that riluzole prolonged median survival of the Irish ALS population by approximately four months, which was shorter than the 7.2 months benefit observed in this study. ${ }^{19}$ Furthermore, the median survival of general neurology clinic patients prescribed riluzole $(\mathrm{n}=149)$ remained 7.2 months shorter than the ALS clinic cohort $(\operatorname{logrank}=16.2, p<0.0001$, figure 3$)$. This indicated that factors other than riluzole contributed to the enhanced survival observed among the ALS clinic cohort.

It was not clear how attendance at a multidisciplinary ALS clinic led to an improved survival. One possible explanation was that the combination of all available therapies (symptomatic and disease modifying) in individual ALS patients was beneficial. Although the prognostic effects of individual treatments (such as PEG enteral nutrition ${ }^{10}$ and riluzole $^{16}$ ) have 
been separately shown to be marginal in clinical trials, the advantage of combining all modalities in the management of individual patients has not been previously evaluated. Non-invasive ventilation is known to prolong survival, ${ }^{20}$ but only a small number of Irish ALS patients received this intervention in this study. Alternatively, McDonald and colleagues have confirmed that ALS patients with psychological distress had a significantly greater risk of death.$^{21}$ It is possible that the frequent interactions with the clinic team and the counselling provided to patients attending the ALS clinic improved quality of life and prolonged survival. This hypothesis was difficult to assess as the Irish ALS Register did not routinely collect data concerning quality of life.

The survival of Irish ALS patients with bulbar onset disease was dramatically improved by attending the ALS clinic-that is, 9.6 months longer than bulbar onset patients followed in general neurology clinics. It is likely that the improved survival among this clinical subgroup reflected the emphasis placed on bulbar function and nutritional status in the multidisciplinary clinic. Despite the observed survival advantage, bulbar onset ALS patients died more rapidly than their limb onset counterparts: median survival of bulbar onset patients attending the ALS clinic was 73 days shorter than limb onset patients attending the ALS clinic. Furthermore, the improvement in prognosis of bulbar onset patients did not entirely account for the improved prognosis of the ALS clinic cohort, as clinic type and bulbar onset disease were independent predictors of prognosis in the multivariate analysis.

In 1999 both the American Academy of Neurology (AAN) and the World Federation of Neurologists (WFN) published guidelines for the optimum management of ALS patients. ${ }^{92}$ The effect of these evidence based or consensus guidelines on ALS survival was unclear. The prognosis of Irish ALS patients attending the ALS clinic before and after 1999 (the date of publication of AAN and WFN guidelines) was statistically similar (logrank test $=0.13, p=0.72$ ). This was not surprising as the AAN guidelines summarised previously published data and the Irish ALS clinic has followed evidence based principles of symptomatic treatment since its inception. ${ }^{6}$ Furthermore, data from the ALS CARE database suggested the majority of ALS patients were managed in accordance with evidence based care even before publication of the AAN parameters. $^{23}$ We conclude that longitudinal studies of specialist ALS clinics over time do not reflect the effect of evidence based management on ALS survival. Instead, a comparison between patients attending ALS clinics and attending general neurology clinics is the most accurate method to quantify the effect of an evidence based approach to ALS management.

In conclusion, using prospectively collected data from the Irish ALS Register over a five year period, we have shown that survival is dramatically improved by attending a multidisciplinary ALS clinic rather than a general neurology clinic. Patients with bulbar onset disease particularly benefited from the active and aggressive management employed in the ALS clinic. Intriguingly, the effect of clinic type was the largest of the four significant covariates, accounting for a $47 \%$ reduction in mortality risk. Although this finding remains to be confirmed in a formal clinical study, the implication of clinic type must be carefully considered in the design of future trials of newer therapeutic agents in ALS patients.

\section{ACKNOWLEDGEMENTS}

We gratefully acknowledge the assistance of the Irish Motor Neuron Disease Association for their support and all doctors who collaborate in recruitment for the Irish ALS Register.

\section{Authors' affiliations}

*B J Traynor, M Alexander, B Corr, O Hardiman, Department of Neurology, Beaumont Hospital, Dublin, Ireland

E Frost, The Irish MND Association, Coleraine House, Dublin, Ireland

*BJT is currently with the Department of Neurology, Massachusetts General Hospital, Boston Ma, USA

\section{REFERENCES}

1 Chancellor AM, Slattery JM, Fraser $\mathrm{H}$, et al. The prognosis of adult-onset motor neuron disease: a prospective study based on the Scottish Motor Neuron Disease Register. J Neurol 1993;240:339-46.

2 Leigh N, Lloyd CM, Ray-Chaudhuri K. Motor neuron disease. In: Wiles $\mathrm{CM}$, ed. Management of neurological disorders. London: BM Publishing Group, 1995: 241-71.

3 World Federation of Neurology. ALS Research Centres, 2002. http://www.wfnals.org/clinics/index.html

4 Aupperle PM, Coyne AC. Primary vs subspecialty care: a structured follow-up of dementia patients and their caregivers. Am J Geriatr Psychiatry 2000;8: 167-70.

5 Traynor BJ, Codd MB, Corr B, et al. Incidence and prevalence of ALS in Ireland, 1995-1997: a population-based study. Neurology 1999;52:504-9.

6 Corr B, Frost E, Traynor BJ, et al. Service provision for patients with ALS/MND: a cost-effective multidisciplinary approach. J Neurol Sci 1998;160 Suppl 1:S141-S145.

7 Brooks BR. El Escorial World Federation of Neurology criteria for the diagnosis of amyotrophic lateral sclerosis. Subcommittee on Motor Neuron Diseases/Amyotrophic Lateral Sclerosis of the World Federation of Neurology Research Group on Neuromuscular Diseases and the El Escorial "Clinical limits of amyotrophic lateral sclerosis" workshop contributors. J Neurol Sci 1994;124 Suppl:96-107.

8 Silani V, Borasio GD. Honesty and hope: announcement of diagnosis in ALS. Neurology 1999;53:S37-S39.

9 Miller RG, Rosenberg JA, Gelinas DF, et al. Practice parameter: the care of the patient with amyotrophic lateral sclerosis (an evidence-based review): report of the Quality Standards Subcommittee of the American Academy of Neurology: ALS Practice Parameters Task Force. Neurology 1999;52:1311-23.

10 Chio A, Finocchiaro E, Meineri P, et al. Safety and factors related to survival after percutaneous endoscopic gastrostomy in ALS. ALS Percutaneous Endoscopic Gastrostomy Study Group. Neurology 1999;53: $1123-5$

11 Thornton FJ, Fotheringham T, Alexander M, et al. Amyotrophic lateral sclerosis: enteral nutrition provision endoscopic or radiologic gastrostomy? Radiology 2002;224:713-7.

12 Microsoft Access. (version 7.0). 1989. Seattle, WA, Microsoft Corporation, 1983.

13 SAS Institute inc. StatView. (version 5.0.1) 1998. Cary, NC, SAS Institute Inc., 1992.

14 Kaplan E, Meier P. Non-parametric estimation from incomplete observations. J Am Stat Assoc 1958;53:457-81.

15 Cox D. Regression models and life-tables. J R Stat Soc B 1972;34:187-220.

16 Lacomblez L, Bensimon G, Leigh PN, et al. Dose-ranging study of riluzole in amyotrophic lateral sclerosis. Amyotrophic Lateral Sclerosis/Riluzole Study Group II. Lancet 1996;347:1425-31.

17 Moore DH. How useful are retrospective studies in evaluating therapeutic interventions? Amyotroph Lateral Scler Other Motor Neuron Disord 2002;3:3

18 Cudkowicz ME, McKenna-Yasek D, Sapp PE, et al. Epidemiology of mutations in superoxide dismutase in amyotrophic lateral sclerosis. Ann Neurol 1997:41:210-21.

19 Traynor BJ, Alexander MA, Corr B, et al. An outcome study of riluzole in Amyotrophic Lateral Sclerosis: a population based study in Ireland, 1996-2000. J Neurol 2003;250:473-9.

20 Aboussouan LS, Khan SU, Meeker DP, et al. Effect of noninvasive positive-pressure ventilation on survival in amyotrophic lateral sclerosis. Ann Intern Med 1997; 127:450-3.

21 McDonald ER, Wiedenfeld SA, Hillel A, et al. Survival in amyotrophic lateral sclerosis. The role of psychological factors. Arch Neurol 1994;51:17-23.

22 Brooks BR. Defining optimal management in ALS: from first symptoms to announcement. Neurology 1999;53:S1-S3.

23 Bradley WG, Anderson F, Bromberg $M$, et al. Current management of ALS: comparison of the ALS CARE Database and the AAN Practice Parameter. The American Academy of Neurology. Neurology $2001 ; 57: 500-4$. 